Supporting Information for:

\title{
Lateral phase separation in lipid-coated microbubbles
}

Mark A. Borden, Gary Martinez, Josette Ricker, Nelly Tsvetkova, Marjorie Longo, Robert Gillies, Paul A. Dayton and Katherine W. Ferrara

\section{NMR standard curve:}

An NMR standard calibration curve was made by plotting the peak area ratio of PEG (3.7 ppm) to $\mathrm{CH}_{2}(1.32 \mathrm{ppm})$ against known concentrations for mixtures containing DSPC and DSPE-PEG2000.

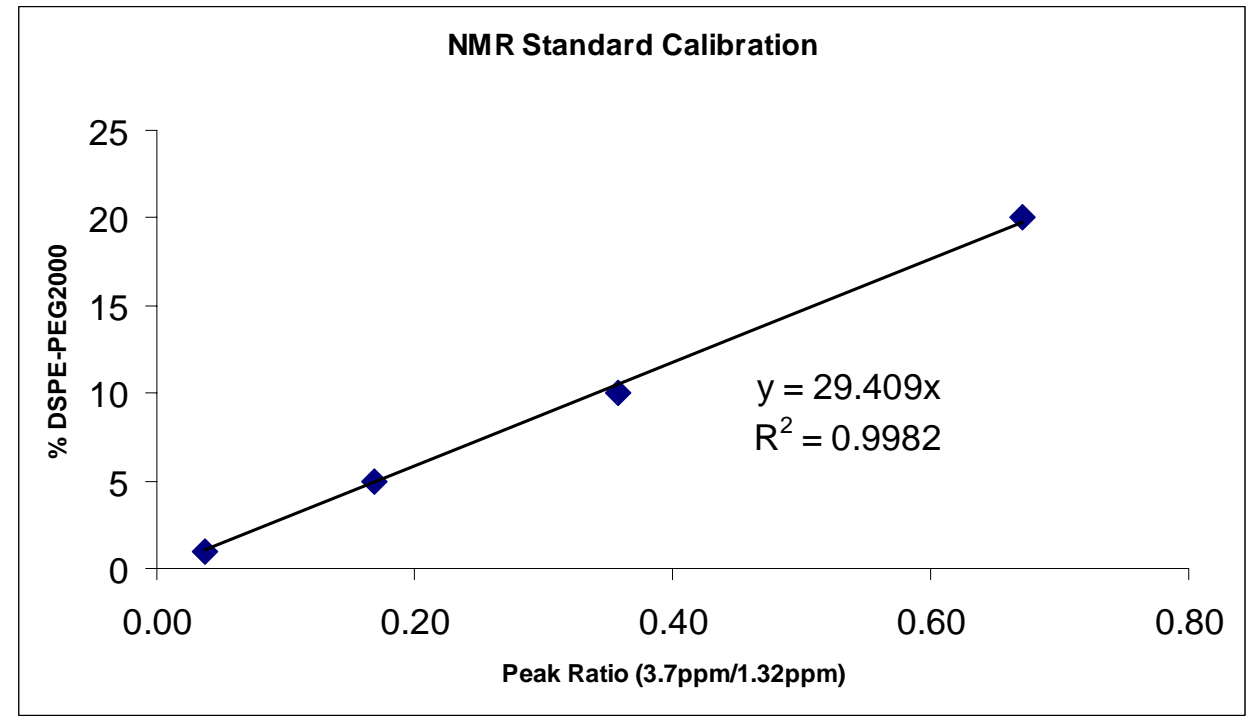

S1. NMR calibration curve.

\section{Anomalous three-phase coexistence video:}

White domains with dark intradomain and interdomain regions were observed occasionally. These domains were highly mobile on the interface. The video shows the domains on a microbubble shell (89.9\% DSPC, 10\% DSPE-PEG2000, 0.1\% DiIC 18 ) moving under flow and Brownian motion. 


\section{Heat treatment to control tethered-ligand distribution:}

Heat treatment was performed following washing and prior to the addition of FITC-NA in a sealed 21-gauge stainless steel tube. Microbubble suspensions were heated to $75^{\circ} \mathrm{C}$ for 3 minutes in order to melt the shell and then cooled by one of the following methods: (1) slow cooling was achieved by submersing the heated tube in a temperature-controlled bath held at 45 ${ }^{\circ} \mathrm{C}$ for 5 minutes; (2) rapid quenching was achieved by submersing the heated tube in an icewater bath held at $3{ }^{\circ} \mathrm{C}$ for 5 minutes. The cooling rates through the lipid transition were simulated with FEMLAB software (Comsol, USA) to be approximately $2-3{ }^{\circ} \mathrm{C} / \mathrm{min}$ for slow cooling and $4000-5000{ }^{\circ} \mathrm{C} / \mathrm{min}$ for rapid quenching. Heat-treated microbubbles were then mixed with FITC-NA $\left(0.5 \mathrm{mg}\right.$ FITC-NA $/ 10^{6}$ microbubbles $\left./ \mathrm{mL}\right)$ and incubated at room temperature for 20 minutes. Excess FITC-NA was removed via a second flotation step and microbubbles were diluted in water for subsequent imaging.

\section{FEMLAB simulation of cooling rates:}

Simulation of the heating and cooling curves was performed using FEMLAB 3.1 software (Comsol, USA). Briefly, an annulus with the dimensions of 21-gauge stainless-steel tubing was constructed to represent the heating/cooling coil. An area with a radius 10-fold greater than the tube outer radius was used to approximate unbounded conditions. The boundary condition at "infinite separation" was set equal to the experimentally measured temperature of the water bath. The initial temperature of the heat transfer zone in the bath was set to the temperature of the entire water bath. The initial temperature of the heating coil was set to the experimental conditions $\left(25^{\circ} \mathrm{C}\right.$ for heating and $75^{\circ} \mathrm{C}$ for cooling). Figure $\mathrm{S} 2$ shows the initial condition and thermal profile after 10 seconds for the rapid quenching experiment. The average temperature at each time point inside the heating coil was determined by integrating over the luminal area. Figure S3 shows the simulated cooling curves and effects on microstructure. 

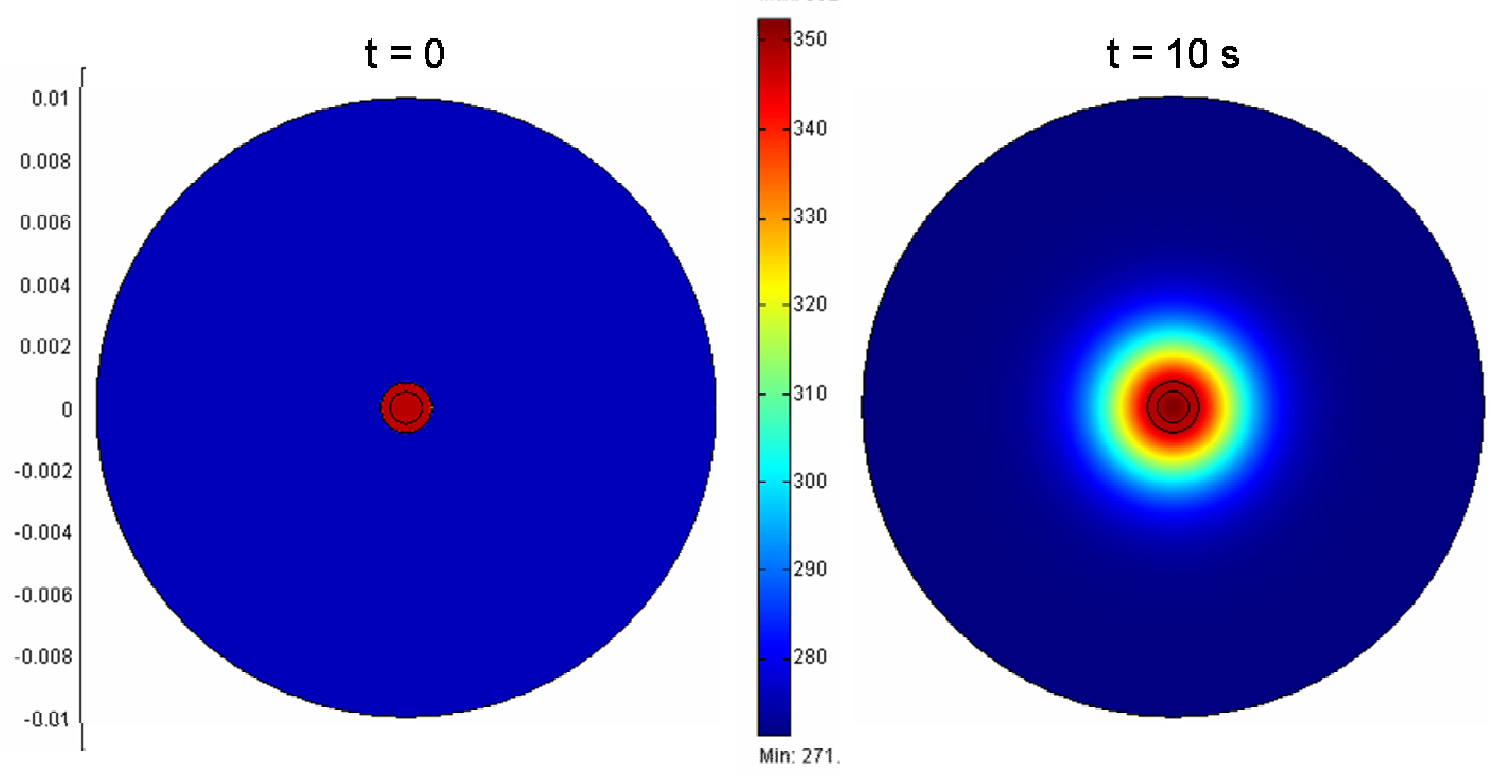

Figure S2. FEMLAB simulation of rapid quenching of a 21-gauge stainless steel heating coil initially at $75{ }^{\circ} \mathrm{C}$ that is instantaneously submerged in water at a set temperature of $3{ }^{\circ} \mathrm{C}$. Left shows initial condition and right shows temperature profile after 10 seconds. Length scale in meters is shown on left, and temperature scale in Kelvin is shown in middle.

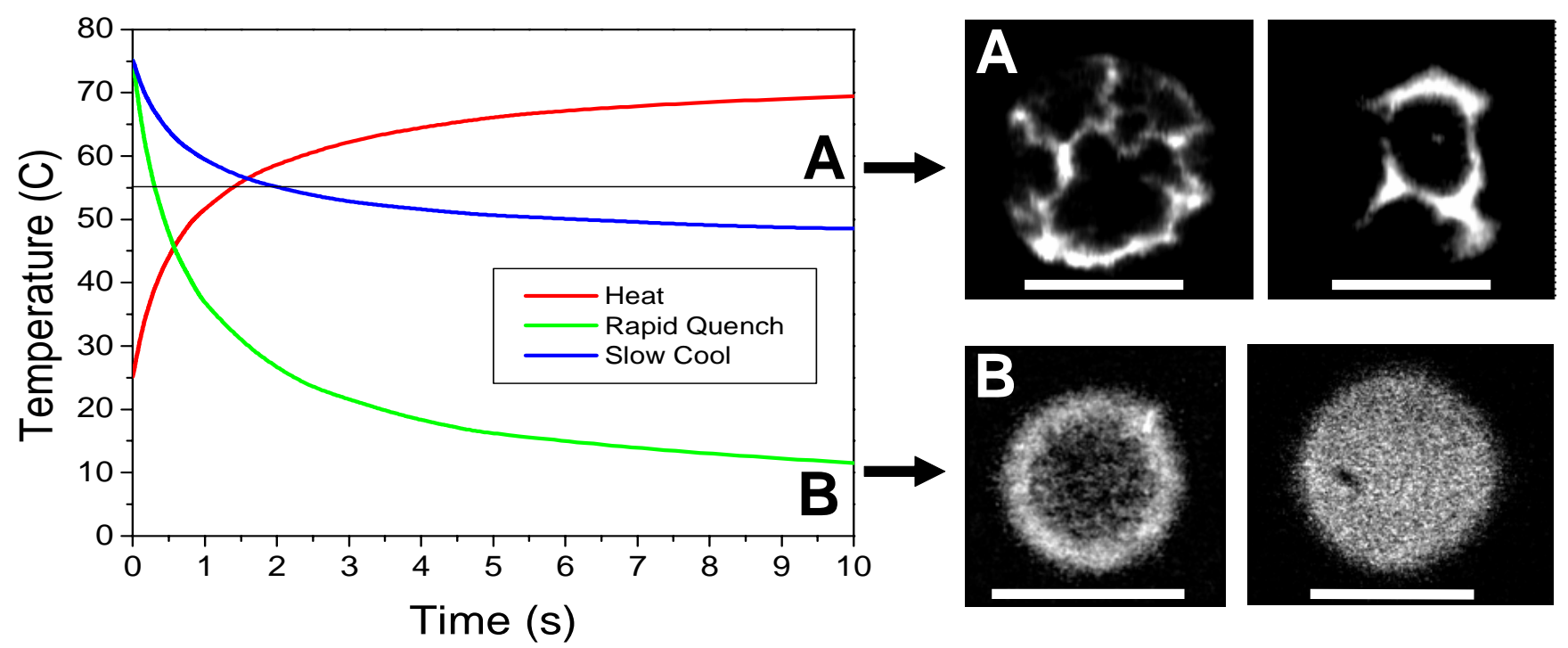

Figure S3. Simulated cooling curves and effect on lipopolymer location. Images were contrast enhanced in PhotoShop. Scale bars equal $10 \mu \mathrm{m}$. 